\title{
A private contributions game for joint replenishment
}

\author{
Evren Körpeoğlu • Alper Şen • Kemal Güler
}

Received: 13 April 2010 / Accepted: 3 November 2011 / Published online: 19 November 2011

(C) Springer-Verlag 2011

\begin{abstract}
We study a non-cooperative game for joint replenishment by $n$ firms that operate under an EOQ-like setting. Each firm decides whether to replenish independently or to participate in joint replenishment, and how much to contribute to joint ordering costs in case of participation. Joint replenishment cycle time is set by an intermediary as the lowest cycle time that can be financed with the private contributions of participating firms. We characterize the behavior and outcomes under undominated Nash equilibria.
\end{abstract}

Keywords Joint replenishment - Economic order quantity -

Non-cooperative games $\cdot$ Private contributions

\section{Introduction}

One of the most fundamental trade-offs in operations is between inventory holding costs and ordering costs as they both change as a function of lot sizes used in production, transportation or procurement. Larger lot sizes lead to higher inventory costs, while smaller lot sizes result in higher ordering costs. Beginning with Harris (1913) study of classical economic order quantity (EOQ), a vast body of literature examined these trade-offs. A second major strand in this literature focused on the joint replenishment problem - exploring opportunities to exploit the economies of scale by consolidating or coordinating replenishment of different items or locations to minimize

\footnotetext{
E. Körpeoğlu · A. Şen (凶)

Department of Industrial Engineering, Bilkent University, Bilkent, 06800 Ankara, Turkey e-mail: alpersen@bilkent.edu.tr

K. Güler

Hewlett-Packard Laboratories, MS 1140, Palo Alto, CA 94304, USA
} 
total ordering and inventory costs. For recent surveys of these two strands of literature the reader is referred to the reviews by Jans and Degraeve (2008) on lot sizing, and by Aksoy and Erengüç (1988) and Khouja and Goyal (2008) on the joint replenishment problem.

When joint replenishment involves a group of items or locations that are not controlled centrally, issues arise regarding sharing of joint costs among the parties. In a series of recent papers, Meca et al. (2004), Hartman and Dror (2007), and Anily and Haviv (2007) analyze cooperative game theory formulations to investigate whether a fair allocation of total costs is possible and if so, how. Meca et al. (2004) show that it is possible to obtain the minimum total joint cost when the firms share their order frequencies. They propose a cost allocation mechanism which distributes the total replenishment cost in proportion to the square of individual order frequencies and show that this allocation is in the core of the game, i.e., no coalition can decrease its costs by defecting from the grand coalition. Minner (2007) studies a similar problem using a bargaining model which has only two firms, excludes inventory holding costs and uses net present value rather than average costs.

In this paper, we study joint replenishment in the context of a non-cooperative game. It is well-known that, in systems where joint decisions have to rely on information reported by the participants, firms may act strategically and misreport their characteristics. In the last two decades, cooperative and non-cooperative game theory have been applied in the analysis of a variety of supply-chain related problems (see Cachon and Netessine 2004; Leng and Parlar 2005; Chinchuluun et al. 2008 for recent comprehensive surveys). Central question of non-cooperative game theory approach is characterization of equilibrium behavior of self-interested players in games where each player's information and strategic options as well as the outcomes that result from each combination of decisions are explicitly specified. Non-cooperative approach enables analyses of several broad sets of research questions: First set concerns analysis of equilibrium outcomes. How do equilibrium outcomes for a given game relate to players' characteristics and how do they vary across environments with different player characteristics? How do equilibrium outcomes of two games compare for a given environment? How do outcomes induced by equilibrium behavior under various alternative game rules perform with respect to a system-optimal solution? Second set deals with questions such as how can one design rules of the non-cooperative interaction to achieve "better" outcomes where the notion of "better" reflects concerns related to system-optimality? As observed by Cachon and Netessine (2004), in decentralized decision making settings obtaining efficiency is the exception rather than the rule.

Game theoretic formulations of the joint replenishment problem seem to have adopted almost exclusively the paradigm of cooperative games with transferable utility. Fiestras-Janeiro et al. (2011) and Dror and Hartman (2011) provide excellent surveys of cooperative game theory applications in centralized inventory management. Despite dozens of papers reviewed in Fiestras-Janeiro et al. (2011) and Dror and Hartman (2011) using cooperative game formulations, non-cooperative analysis of joint inventory problems is still in its infancy with many interesting problems that remain to be explored using the machinery of non-cooperative game theory. In fact, 
Bauso et al. (2008) and Meca et al. (2003) are the only two exceptions that look at the joint replenishment problem from a non-cooperative point of view.

Bauso et al. (2008) study a finite horizon, periodic setting in which multiple firms need to determine their order quantities in each period to satisfy their deterministic, time varying customer demands. The fixed order cost is shared among multiple firms that order in the same period. Bauso et al. (2008) show that this game admits a set of pure strategy Nash equilibria, one of which is Pareto optimal. The authors present a consensus protocol that leads the firms converge to one of Nash equilibria, but not necessarily a Pareto optimal one.

Meca et al. (2003) (MGB in the sequel) is more closely related to our work. MGB studies a non-cooperative reporting game where stand-alone order frequencies of the firms are observable but not verifiable. Each firm reports an order frequency (that may be different from its true order frequency) and the joint order frequency is determined to minimize the total joint costs based on all reports. Each firm incurs holding cost individually and pays a share of the joint replenishment cost in proportion to the squares of reported order frequencies. MGB shows that, while this rule leads to core allocations under cooperative formulations, it entails significant misreporting and inefficient joint decisions in a non-cooperative framework.

We consider $n$ firms with arbitrary inventory holding cost and demand rates. The firms' characteristics are common knowledge, but they are not verifiable. Each firm decides whether to participate in joint replenishment or to replenish independently, and each participating firm reports the level of his private contribution to the joint ordering costs. An intermediary determines the joint cycle time. The intermediary selects the lowest joint cycle time that can be financed with the participating firms' contributions.

The game we study differs from the one in MGB in several important ways with respect to messages the firms can use and with respect to the outcome functions that specify how joint decisions and individual cost shares are determined based on firms' messages. MGB considers a game where firms' messages are their stand-alone order frequencies. We study a game where each firm decides whether to replenish independently or to participate in joint replenishment and, if he participates, reports the level of his private contribution to the joint ordering cost. With respect to the outcomes functions, while the joint frequency decision in MGB is the efficient joint decision assuming truthful reporting by the firms, in our game joint replenishment frequency is determined to cover the replenishment cost based on the private contributions of participating firms. A participating firm's replenishment cost depends on all the reports through a proportional sharing rule in MGB, whereas, in our setting, it is determined by his report directly.

We find that equilibrium behavior and outcomes are determined by a simple property of joint replenishment environment: If there is a single firm with the lowest stand-alone cycle time, then there is a unique undominated Nash equilibrium. Otherwise, that is, if there are multiple firms with the lowest stand-alone cycle time, there are multiple equilibria. However, the only indeterminacy caused by multiple equilibria concerns how the firms with the lowest stand-alone cycle time share a given aggregate replenishment cost (which is unique across all equilibria). Aggregate contributions, joint cycle time, aggregate cost rates, as well as cost rates for firms whose stand-alone cycle times are 
higher than the lowest stand-alone cycle time are all unique. Furthermore, the unique equilibrium is such that all firms participate in joint replenishment. Equilibrium joint cycle time is equal to the lowest stand-alone cycle time. In general, equilibrium contributions involve substantial free-riding as in general public good problems (Bergstrom et al. 1986).

\section{The model and preliminaries}

We consider a stylized EOQ environment with a set of firms $N=\{1, \ldots, n\}$. Demand rate for firm $j$ is constant and deterministic at $\beta_{j}$ per unit of time. Time rate of inventory holding cost for firm $j$ is $\lambda_{j}$ per unit. Major ordering cost is fixed at $\kappa$ per order regardless of order size. We assume minor ordering costs (ordering costs associated with firms included in an order) are zero. ${ }^{1}$ Although each firm is characterized by two parameters $\left(\lambda_{j}, \beta_{j}\right)$, an alternative representation $\left(\alpha_{j}, \beta_{j}\right)$, obtained by a re-parametrization where $\alpha_{j}=\lambda_{j} \beta_{j}$, will be convenient in all the settings that we consider below. For lack of a more natural term, we refer to the parameter $\alpha$ as the adjusted demand rate. We assume a strictly positive lower bound, $\underline{\alpha}>0$, for the adjusted demand rates, so that $\alpha_{j} \geq \underline{\alpha}$ for all $j \in N$ to rule out trivial replenishment environments where either the demand rate or the holding cost rate is zero.

For $j \in N$, the ratio

$$
\theta_{j}=\alpha_{j} / \sum_{k \in N} \alpha_{k}
$$

will prove useful to simplify some comparisons in the sequel.

In a stylized replenishment problem the objective is to minimize the total cost rate, denoted $C$, i.e., the sum of replenishment cost rate $(R)$ and holding cost rate $(H): C=R+H$. The decision variable can be taken as order cycle time, $t$, or order frequency, $f=1 / t$ (number of orders per time unit). We take cycle time as the decision variable in the sequel.

We use upper-case letters, $N, M, L$ etc., to refer to sets of firms, and use the lowercase version of the same letter for the cardinality of a set. The letters $i, j, k$ are used for firm indices. We label the firms so that $\alpha_{1} \leq \alpha_{2} \leq \cdots \leq \alpha_{n}$. This ordering of firm indices is retained for subsets of $N$. For $M \subseteq N$, denote the set of firms in $M$ with the highest values of the parameter $\alpha$ by $L(M)=\left\{j \in M \mid \alpha_{j} \geq \alpha_{i}\right.$ for all $\left.i \in M\right\}$.

We denote vectors by lower-case letters in bold typeface. For a generic $m$-tuple $\boldsymbol{x}=\left(x_{1}, \ldots, x_{m}\right)$ and $j \in\{1, \ldots, m\}$, the notation $\left(y, \boldsymbol{x}_{-j}\right)$ stands for the vector $\boldsymbol{x}$ with its $j$ th entry $x_{j}$ replaced by $y$, and the $(m-1)$-tuple $\boldsymbol{x}_{-j}$ stands for the vector $\boldsymbol{x}$ with its $j$ th entry $x_{j}$ removed.

\footnotetext{
${ }^{1}$ Following a stylized EOQ environment, such as one given in Zipkin (2000, §3.2), it is assumed that the outside supplier that replenishes the orders has no capacity restrictions, delivers the complete order at once after a deterministic lead time and has perfect yield. It is also assumed that the outside supplier is not a strategic player. The firms aim to minimize their long-run average costs over time and backorders are not allowed.
} 
For an endogenous variable $X$, by $X_{M}^{a}$ we refer to the value of $X$ when the set of firms is $M$ and replenishment operations are governed by $a \in\{c, d, g\}$, where $c$ stands for centralized, $d$ stands for decentralized (or independent) replenishment, and $g$ stands for joint replenishment under rules of the non-cooperative game $g$. For instance, $T_{M}^{c}$ is the joint cycle time of the firms in $M$ when replenishment is centralized. When the set $M$ is a singleton, e.g., $M=\{j\}$, we use $X_{j}^{a}$ instead of $X_{\{j\}}^{a}$. When we need to refer to the value of an endogenous variable $X_{M}^{a}$ faced by firm $j \in M$ we use $X_{M j}^{a}$. Thus, for instance, $R_{M j}^{c}$ is the replenishment cost faced by firm $j \in M$ when the firms in $M$ replenish jointly.

The vector $e=(N, \kappa, \boldsymbol{\alpha}, \boldsymbol{\beta})$ summarizes the essential data of the inventory environment.

\subsection{Independent (decentralized) replenishment}

When the replenishment of the items is controlled by firms operating independently, firm $j$ 's total cost rate $\left(C_{j}\right)$ is the sum of replenishment cost rate $\left(R_{j}\right)$ and the holding cost rate $\left(H_{j}\right)$ :

$$
C_{j}(t)=R_{j}(t)+H_{j}(t)=\frac{\kappa}{t}+\frac{t}{2} \alpha_{j}
$$

It is well known that firm $j$ 's optimal cycle time is $T_{j}^{d}=\sqrt{2 \kappa / \alpha_{j}}$. Hence, optimal frequency and optimal order quantity are $F_{j}^{d}=\sqrt{\alpha_{j} / 2 \kappa}$ and $Q_{j}^{d}=\beta_{j} \sqrt{2 \kappa / \alpha_{j}}$, respectively. This leads to a replenishment cost rate of $R_{j}^{d}=\sqrt{\kappa \alpha_{j} / 2}$. Firm $j$ 's holding cost rate is also $H_{j}^{d}=\sqrt{\kappa \alpha_{j} / 2}$. Thus firm $j$ 's total cost per unit of time is $C_{j}^{d}=\sqrt{2 \kappa \alpha_{j}}$. The aggregate total cost rates for $n$ firms under independent replenishment are $C_{N}^{d}=\sum_{k \in N} \sqrt{2 \kappa \alpha_{k}}$, and $R_{N}^{d}=H_{N}^{d}=\sum_{k \in N} \sqrt{\kappa \alpha_{k} / 2}$.

\subsection{Joint (centralized) replenishment}

Efficient joint replenishment requires the replenishment decisions to be taken centrally to minimize the aggregate total cost. It is well known that when there are no minor setup costs, all firms will be replenished in each cycle leading to a common cycle time (see, for example, Meca et al. 2004). The aggregate cost for $n$ firms as function of the common cycle time $t$ can be written as

$$
C_{N}(t)=R_{N}(t)+H_{N}(t)=\frac{\kappa}{t}+\frac{t}{2} \sum_{k \in N} \alpha_{k} .
$$

The optimal cycle time and the corresponding optimal frequency are $T_{N}^{c}=$ $\sqrt{2 \kappa / \sum_{k \in N} \alpha_{k}}$ and $F_{N}^{c}=\sqrt{\sum_{k \in N} \alpha_{k} / 2 \kappa}$, respectively. Then, the optimal cost rates are $C_{N}^{c}=\sqrt{2 \kappa \sum_{k \in N} \alpha_{k}}$, and $R_{N}^{c}=H_{N}^{c}=C_{N}^{c} / 2$. At each cycle, firm $j$ orders $Q_{N j}^{c}=\beta_{j} T_{N}^{c}$. 


\subsection{Comparing joint and independent replenishment}

With $\theta_{j}=\alpha_{j} / \sum_{k \in N} \alpha_{k}$, optimal cycle times, order frequencies and holding cost rates under joint and independent ordering are related by:

$$
T_{N}^{c}=\sqrt{\theta_{j}} T_{j}^{d}, F_{N}^{c}=F_{j}^{d} / \sqrt{\theta_{j}} \text { and } H_{N j}^{c}=\sqrt{\theta_{j}} H_{j}^{d}
$$

On the other hand, comparison of replenishment costs, hence of total costs, incurred by an individual firm under joint replenishment and independent replenishment depends on how the joint costs are shared. If the joint ordering cost is allocated proportionally so that firm $j$ pays $\theta_{j}$ of the order cost, firm $j$ 's replenishment cost rate and total cost rate under joint ordering and independent ordering are also related by the factor $\theta_{j}: R_{N j}^{c}=\sqrt{\theta_{j}} R_{j}^{d}$ and $C_{N j}^{c}=\sqrt{\theta_{j}} C_{j}^{d}$.

Straightforward comparison of the cycle times under independent and joint replenishment yield:

$$
T_{1}^{d} \geq T_{2}^{d} \geq \cdots \geq T_{n}^{d}=\frac{1}{\sqrt{\theta_{n}}} T_{N}^{c}>T_{N}^{c} .
$$

Similarly, comparing aggregate total costs under independent and joint replenishment regimes we get:

$$
C_{N}^{d}=\left(\sum_{k \in N} \sqrt{\theta_{k}}\right) C_{N}^{c}>C_{N}^{c}
$$

Potential cost saving from joint replenishment in relative terms is governed by the vector $\boldsymbol{\theta}$ :

$$
\frac{C_{N}^{d}-C_{N}^{c}}{C_{N}^{c}}=\sum_{k \in N} \sqrt{\theta_{k}}-1 .
$$

As this measure is strictly concave in $\boldsymbol{\theta}$, it is maximized when $\theta_{i}=1 / n$ for all $i \in N$ (i.e., when all firms have a common $\alpha$ ), and minimized when $\theta_{n}=1$. When $\theta_{i}=1 / n$, the measure simplifies to $\sqrt{n}-1$.

\subsection{MGB: a direct mechanism for joint replenishment}

MGB considers a a direct mechanism where the message set of each player coincides with the set of all possible characteristics a player may have and the outcome function assigns the core allocation for the environment reported by the players. Specifically, the firms' stand-alone order frequencies are used as the message space - each firm reports an order frequency that may be different from its true order frequency. Each firm $j$ either reports a positive frequency $f_{j}$ and joins the coalition for joint replenishment or reports $f_{j}=0$ and orders independently. Each firm incurs holding cost individually and the joint replenishment cost is allocated by a proportional sharing rule whereby firms 
share the joint ordering cost in proportion to the squares of reported order frequencies. For any profile of reported frequencies $\left(f_{1}, \ldots, f_{n}\right)$, if the number of firms reporting strictly positive frequencies is two or less, all firms replenish independently. With two or more firms reporting positive frequencies, the joint frequency is determined as the efficient frequency for the reported stand-alone frequencies. However, as MGB find, equilibrium behavior in this game entails significant misreporting. The authors show that the game has multiple equilibria. The strategy profile $\left(f_{1}, \ldots, f_{n}\right)=(0, \ldots, 0)$ is always an equilibrium resulting in all firms replenishing independently. An equilibrium (dubbed "constructive equilibrium" by the authors) in which all firms participate in joint replenishment exists if, and only if, the firms are sufficiently homogeneous, i.e., if and only if

$$
\theta_{n}<\frac{2}{2 n-1}
$$

With straightforward translation of MGB's notation to our setting, when a constructive equilibrium exists, it yields the following cycle time and aggregate total cost:

$$
T_{N}^{M G B}=\sqrt{\frac{2 \kappa(2 n-1)}{\sum_{k \in N} \alpha_{k}}}=\sqrt{2 n-1} T_{N}^{c},
$$

and

$$
C_{N}^{M G B}=\sqrt{\frac{2 \kappa n^{2} \sum_{k \in N} \alpha_{k}}{(2 n-1)}}=\frac{n}{\sqrt{2 n-1}} C_{N}^{c} .
$$

Although the rules of the MGB game would give rise to core allocations with desirable efficiency and fairness properties under truthful reporting, under non-cooperative behavior, we get substantial efficiency loss. In the remainder of this paper, we investigate the equilibrium outcomes and whether more efficient outcomes can be achieved under an alternative set of rules governing the interaction of the potential participants in joint replenishment.

\section{Private contribution games for joint replenishment}

The participation-contribution game we consider has the following elements: each firm makes two decisions: (1) whether to replenish independently or to participate in joint replenishment, and (2) how much to contribute to joint ordering cost in case of participation. We assume a small but strictly positive lower bound $\delta$ on the contributions for participation in joint replenishment. ${ }^{2}$ Specifically, we assume

$$
0<\delta<\bar{\delta}=\sqrt{\kappa \underline{\alpha} / 2} / n \text {. }
$$

\footnotetext{
2 The assumed bound on $\delta$ is tighter than needed for the characterization results we present to hold. However, assuming weaker bounds amounts to assuming that the intermediary has more detailed information
} 
Formally, the strategy set of players is represented by non-negative real numbers, $\mathcal{M}=\mathbb{R}_{+}$. A message $r_{j}$ from player $j$ codes the participation and contribution decisions of firm $j$ as follows: If $r_{j}<\delta$, firm $j$ stays out and replenishes independently, if $r_{j} \geq \delta$, it represents time rate of private contribution to the joint ordering cost.

We denote the vector of messages of the $n$ firms $r=\left(r_{1}, \ldots, r_{n}\right)$. The set of firms who selected to participate in joint replenishment are denoted by $M(\boldsymbol{r})=\left\{i \in N \mid r_{i} \geq\right.$ $\delta\}$. For $M \subseteq N$, the tuple $\boldsymbol{r}_{M}$ collects the components of the vector $\boldsymbol{r}$ that correspond to the coordinates in $M$.

Players move simultaneously and each decides his message. For any message profile $\boldsymbol{r}$, the intermediary selects the lowest cycle time that can be financed with the aggregate collection from the participating firms $\sum_{k \in M(r)} r_{k}$, i.e.,

$$
\tau(\boldsymbol{r})=\frac{\kappa}{\sum_{k \in M(\boldsymbol{r})} r_{k}} .
$$

Implicit in the intermediary's decision rule is an assumption regarding the structure of information held by the firms and the intermediary. The intermediary cannot make use of firm-specific information beyond the contribution decisions reported by individual firms. To be able to decide the joint cycle time, she also needs to know the fixed ordering $\cos t \kappa$, in addition to the private contributions from the participating firms (and, hence, the set of participating firms).

Then for given $n$-tuple of messages $\boldsymbol{r}$, the outcome is determined as follows: If $r_{j}<\delta$, firm $j$ replenishes independently, and his cost is $C_{j}^{d}$. All firms in $M(\boldsymbol{r})$ replenish together with joint cycle time $\tau(\boldsymbol{r})$ selected by the intermediary, and firm $j \in M(\boldsymbol{r})$ pays $r_{j}$ per unit of time as his contribution to joint replenishment cost. ${ }^{3} \mathrm{~A}$ participating firm's replenishment cost rate $\left(R_{j}\right)$ is determined directly by his private contribution, $R_{j}=r_{j}$, while his holding cost rate $\left(H_{j}\right)$ depends on the joint cycle time, $H_{j}=\alpha_{j} \tau(\boldsymbol{r}) / 2$.

The rules of the private contributions mechanism are common knowledge. The parameters of the replenishment environment, i.e., the elements of the list $(\kappa, \boldsymbol{\alpha}, \boldsymbol{\beta})$, are also common knowledge among the firms (but not verifiable).

We can now state the total cost per unit of time for firm $j$, denoted $\phi_{j}$, as a function of the firms' messages:

$$
\phi_{j}(\boldsymbol{r})= \begin{cases}\sqrt{2 \kappa \alpha_{j}} & \text { if } r_{j}<\delta \\ r_{j}+\frac{1}{2} \alpha_{j} \tau(\boldsymbol{r}) & \text { if } r_{j} \geq \delta\end{cases}
$$

\footnotetext{
Foonote 2 continued

on the firm-specific details of the replenishment environment, specifically, about the parameter vector $\boldsymbol{\alpha}$. The bound $\bar{\delta}$ involves minimal information about the environment, namely, $n, \kappa$ and $\underline{\alpha}$. Furthermore, under weaker bounds, equilibrium characterization involves complications with many cases and subcases to be considered. If the minimum contribution $\delta$ were to be completely independent of the parameter vector $\boldsymbol{\alpha}$, one could always find replenishment environments where, in the unique equilibrium, no firm participates in joint replenishment.

3 Operationally, the payments for replenishment can be made at the time of the ordering with firm $j \in M(r)$ paying $r_{j} \tau(\boldsymbol{r})$ independent of his order size. Or, firm $j$ can pay a flow of $r_{j}$ per unit of time without any additional payment at replenishment points.
} 
Taking other firms' strategies $\boldsymbol{r}_{-j}$ as given, firm $j$ 's decision problem is

$$
\min _{r_{j}} \phi_{j}(\boldsymbol{r})
$$

and his best response function, denoted $\rho_{j}$ is

$$
\rho_{j}\left(\boldsymbol{r}_{-j}\right)=\arg \min _{r_{j}} \phi_{j}\left(r_{j}, \boldsymbol{r}_{-j}\right)
$$

A Nash equilibrium is a profile $\boldsymbol{r}^{*}=\left(r_{1}^{*}, \ldots, r_{n}^{*}\right)$ such that $r_{j}^{*}=\rho_{j}\left(\boldsymbol{r}_{-j}^{*}\right)$ for all $j \in N$. A strategy $y$ is said to strictly dominate strategy $x$ for player $j$ if $\phi_{j}\left(y, \boldsymbol{r}_{-j}\right)<$ $\phi_{j}\left(x, \boldsymbol{r}_{-j}\right)$ for all $(n-1)$-tuple $\boldsymbol{r}_{-j}$ of other players' strategies. A strategy $y$ is said to weakly dominate strategy $x$ for player $j$ if $\phi_{j}\left(y, \boldsymbol{r}_{-j}\right) \leq \phi_{j}\left(x, \boldsymbol{r}_{-j}\right)$ for all $(n-1)$ tuple $\boldsymbol{r}_{-j}$ of other players' strategies, with strict inequality for at least one $\boldsymbol{r}_{-j}$. A strategy $x$ is said to be an undominated strategy for player $j$ if there is no other strategy that weakly dominates it. A profile of strategies $\boldsymbol{r}^{*}=\left(r_{1}^{*}, \ldots, r_{m}^{*}\right)$ is a Nash equilibrium in undominated strategies or undominated Nash equilibrium (UNE) if $r_{j}^{*}$ is an undominated strategy for player $j$.

Substituting the rule that determines the joint cycle time, firm $j$ 's total cost per unit becomes:

$$
\phi_{j}(\boldsymbol{r})=\phi_{j}\left(r_{j}, \boldsymbol{r}_{-j}\right)= \begin{cases}\sqrt{2 \kappa \alpha_{j}} & \text { if } r_{j}<\delta \\ r_{j}+\frac{\kappa \alpha_{j}}{2\left(r_{j}+\sum_{k \in M(r) \backslash\{j\}} r_{k}\right)} & \text { if } r_{j} \geq \delta\end{cases}
$$

Before we proceed, we collect several observations each with simple proofs.

Claim 1 For all replenishment environments, any strategy profile $\boldsymbol{r}$ with $M(\boldsymbol{r})=\emptyset$, that is, $r_{j}<\delta$ for all $j \in N$, is a Nash equilibrium.

Proof: Given that other firms are not participating, no strategy $r \geq \delta$ yields a better cost to a player than the cost he gets from independent replenishment.

Claim 2 If $\boldsymbol{r}$ is a Nash equilibrium, then $M(\boldsymbol{r}) \in\{\varnothing, N\}$. That is, unless $\boldsymbol{r}$ yields full participation or no participation, it cannot be a Nash equilibrium.

Proof: Suppose $M(\boldsymbol{r})$ is a non-empty strict subset of $N$, and consider a firm $j \in$ $N \backslash M(\boldsymbol{r})$. Since $j \notin M(\boldsymbol{r})$ player's cost is $C_{j}^{d}$. Let $w=\sum_{k \in M(\boldsymbol{r})} r_{k}$. Since $M(\boldsymbol{r}) \neq \emptyset$, it must be that $w>0$. If player $j$ deviates from $r_{j}$ to $R_{j}^{d}$ he gets

$$
\begin{aligned}
\phi_{j}\left(R_{j}^{d}, \boldsymbol{r}_{-j}\right) & =R_{j}^{d}+\frac{\kappa \alpha_{j}}{2\left(R_{j}^{d}+w\right)}<R_{j}^{d}+\frac{\kappa \alpha_{j}}{2\left(R_{j}^{d}\right)} \\
& =2 R_{j}^{d}=C_{j}^{d}=\phi_{j}\left(r_{j}, \boldsymbol{r}_{-j}\right) .
\end{aligned}
$$

where the inequality follows from the fact that $w>0$, and subsequent equalities follow from the facts $R_{j}^{d}=\sqrt{\kappa \alpha_{j} / 2}$ and $C_{j}^{d}=2 R_{j}^{d}$.

Claim 3 Any strategy $\hat{r}_{j}<\delta$ is weakly dominated by the strategy $\tilde{r}_{j}=R_{j}^{d}$. 
Proof: This follows from observing that the cost strategy $\hat{r}_{j}$ yields is exactly $C_{j}^{d}=$ $\sqrt{2 \kappa \alpha_{j}}$ while the strategy $\tilde{r}_{j}$ yields a cost that is equal to $C_{j}^{d}$ when other players all stay out of joint replenishment, and a cost that is strictly better in all other cases.

Claim 4 Any strategy $\hat{r}_{j}>R_{j}^{d}$ is strictly dominated by the strategy $\tilde{r}_{j}=R_{j}^{d}$.

Proof: Let $w=\sum_{k \in M(r) \backslash\{j\}} r_{k}$. Since $\phi_{j}\left(r, \boldsymbol{r}_{-j}\right)=\Phi_{j}(r, w)=r+\frac{\kappa \alpha_{j}}{2(r+w)}$ is strictly convex in $r$, and since the cross-partial $\frac{\partial^{2} \Phi_{j}}{\partial r \partial w}=\frac{\kappa \alpha}{(r+w)^{3}}>0$, it follows from the Implicit Function Theorem that $r(w)=\arg \min _{r} \Phi_{j}(r, w)$ is unique and strictly decreasing in $w$. Thus, for $w>0$, we get

$$
r(w)<r(0)=R_{j}^{d}<\hat{r}_{j},
$$

which implies, because $\Phi_{j}(r, w)$ is strictly convex in $r$, that

$$
\Phi_{j}(r(w), w)<\Phi_{j}\left(R_{j}^{d}, w\right)<\Phi_{j}\left(\hat{r}_{j}, w\right)
$$

Hence $R_{j}^{d}$ strictly dominates $\hat{r}_{j}$.

From Claims 3 and 4 it follows that the set of undominated strategies is the interval $\left[\delta, R_{j}^{d}\right]$. From claims 1 and 3 it follows that if a Nash equilibrium in undominated strategies exists, it involves full participation in joint replenishment. We record these observations in the following proposition.

Proposition 1 If $\boldsymbol{r}^{*}$ is a Nash equilibrium in undominated strategies, then

1. $M\left(\boldsymbol{r}^{*}\right)=N$ and

2. $r_{j}^{*} \in\left[\delta, R_{j}^{d}\right]$.

It remains to characterize the finer details of structure of best response functions and the equilibrium contribution levels. The foregoing observations greatly simplify our task in that they allow us to focus on the second-piece of the cost function and take $M(\boldsymbol{r})=N$ in the remainder of our investigation. That is,

$$
\rho_{j}\left(\boldsymbol{r}_{-j}\right)=\arg \min _{r_{j} \geq \delta} r_{j}+\frac{\kappa \alpha_{j}}{2\left(r_{j}+\sum_{k \in N \backslash\{j\}} r_{k}\right)} .
$$

In order to find the best response of firm $j$, we take the derivative of $\phi_{j}\left(r_{j}, \boldsymbol{r}_{-j}\right)$ with respect to $r_{j}$ and re-arrange terms:

$$
\frac{\partial \phi_{j}}{\partial r_{j}}=1-\frac{\kappa \alpha_{j}}{2\left(r_{j}+\sum_{k \in N \backslash\{j\}} r_{k}\right)^{2}} .
$$

Solving $\partial \phi_{j} / \partial r_{j}=0$, and incorporating the minimum contribution requirement, we get:

$$
\rho_{j}\left(\boldsymbol{r}_{-j}\right)=\max \left\{\delta, \sqrt{\frac{\kappa \alpha_{j}}{2}}-\sum_{k \in N \backslash\{j\}} r_{k}\right\} .
$$


Rewriting (13), we obtain:

$$
\rho_{j}\left(\boldsymbol{r}_{-j}\right)= \begin{cases}R_{j}^{d}-\sum_{k \in N \backslash\{j\}} r_{k}, & \text { if } \sum_{k \in N \backslash\{j\}} r_{k} \leq R_{j}^{d}-\delta, \\ \delta, & \text { if } \sum_{k \in N \backslash\{j\}} r_{k}>R_{j}^{d}-\delta,\end{cases}
$$

which states that firm $j$ 's best response is to contribute such that the aggregate contributions are equal to firm $j$ 's stand-alone ordering cost, if the aggregate contributions of other firms are less than firm $j$ 's stand-alone ordering cost minus the minimum required amount, and contribute the minimum required amount, otherwise. If firms in $N \backslash\{j\}$ each contributed $\delta$, firm $j$ 's best response would be to contribute $R_{j}^{d}-(n-1) \delta$ leading to an aggregate contribution of $R_{j}^{d}$ from $n$ firms and a cycle time $\tau_{N}=T_{j}^{d}$. Note that $R_{j}^{d}-(n-1) \delta=\sqrt{\kappa \alpha_{j} / 2}-(n-1) \delta$ is strictly larger than $\delta$ since $\delta<\sqrt{\kappa \underline{\alpha} / 2} / n \leq \sqrt{\kappa \underline{\alpha} / 2} / n$ and $\underline{\alpha} \leq \alpha_{j}$. For every dollar of contribution from firms in $N \backslash\{j\}$, firm $j$ reduces his contribution dollar for dollar until he reaches the minimum required contribution.

The first pieces of the piecewise-linear best response functions in (14) have the same slope (i.e., -1$)$ and their intercepts $\left(R_{j}^{d}\right.$ for firm $\left.j\right)$ are ordered. Equilibrium lies in the intersection of best response functions (i.e., solution of $r_{j}=\rho_{j}\left(\sum_{k \in N \backslash\{j\}} r_{k}\right)$ for all $j$ ).

In equilibrium, aggregate contributions must be $R_{n}^{d}=\max _{j \in N} R_{j}^{d}$. Otherwise, if aggregate contributions were such that $R_{n}^{d}-\sum_{j \in N} r_{j}=R_{n}^{d}-\sum_{j \in N \backslash\{m\}} r_{j}-r_{n}=$ $\Delta>0$, firm $n$ would increase his contribution from $r_{n}$ to $r_{n}+\Delta$, and using (9), this would lead his total cost to decrease from $2 R_{n}^{d}+\Delta^{2} /\left(R_{n}^{d}-\Delta\right)-\sum_{j \in N \backslash\{n\}} r_{j}$ to $2 R_{n}^{d}-\sum_{j \in N \backslash\{n\}} r_{j}$.

In the next proposition we provide a complete characterization of the Nash equilibria in undominated strategies, followed by a formal proof.

Proposition 2 In the private contributions joint replenishment game with $\delta<$ $\sqrt{\kappa \underline{\alpha} / 2} / n$ :

1. A profile of strategies $\boldsymbol{r}^{*}=\left(r_{1}^{*}, \ldots, r_{n-\ell}^{*}, r_{n-\ell+1}^{*}, \ldots r_{n}^{*}\right)$ is a Nash equilibrium in undominated strategies (UNE) if and only if

(a) $r_{j}^{*}=\delta$ for all $j \in N \backslash L(N)$, and

(b) $\left(r_{n-\ell+1}^{*}, \ldots r_{n}^{*}\right) \in$

$\left\{\boldsymbol{x} \in \mathbb{R}^{\ell} \mid x_{i} \geq \delta\right.$, for $i=1, \ldots, \ell$, and $\left.\sum_{i \in L(N)} x_{i}=\sqrt{\kappa \alpha_{n} / 2}-(n-\ell) \delta\right\}$.

2. The equilibrium is unique if and only if $L(N)$ is a singleton, i.e., if and only if $\alpha_{n-1}<\alpha_{n}$. In the unique equilibrium, $r_{j}^{*}=\delta$ for $j=1, \ldots, n-1$ and $r_{n}^{*}=R_{n}^{d}-(n-1) \delta$.

3. In all equilibria, aggregate contributions and the joint cycle time are unique:

(a) Aggregate contributions: $\sum_{k \in N} r_{k}^{*}=\sqrt{\kappa \alpha_{n} / 2}=R_{n}^{d}$

(b) Cycle time: $T_{N}^{g}=\tau_{N}\left(\boldsymbol{r}^{*}\right)=\sqrt{2 \kappa / \alpha_{n}}=T_{n}^{d}$.

4. Equilibrium aggregate cost rates are also unique:

(a) Aggregate replenishment cost: $R_{N}^{g}=\sum_{k \in N} r_{k}^{*}=\sqrt{\kappa \alpha_{n} / 2}=R_{n}^{d}$

(b) Aggregate holding cost: $H_{N}^{g}=\left(\sum_{k \in N} \alpha_{k}\right) \sqrt{\kappa / 2 \alpha_{n}}$ 
(c) Aggregate total cost: $C_{N}^{g}=\sqrt{\kappa / 2 \alpha_{n}}\left(\alpha_{n}+\sum_{k \in N} \alpha_{k}\right)$.

5. In equilibrium firm $j$ faces the following cost rates

(a) Replenishment cost: $R_{N j}^{g}=\delta$ if $j \in N \backslash L(N)$, and $R_{N j}^{g} \in\left[\delta, R_{n}^{d}-(n-1) \delta\right]$ if $j \in L(N)$.

(b) Holding cost: $H_{N j}^{g}=\alpha_{j} \sqrt{\kappa / 2 \alpha_{n}}$

(c) Total cost: $C_{N j}^{g}=\delta+\alpha_{j} \sqrt{\frac{\kappa}{2 \alpha_{n}}}$ if $j \in N \backslash L(N)$, and $C_{N j}^{g} \in\left[\sqrt{\kappa \alpha_{n} / 2}+\right.$ $\left.\delta, \sqrt{\kappa \alpha_{n} / 2}+R_{n}^{d}-(n-1) \delta\right]$ if $j \in L(N)$.

Proof For part 1 we provide detailed arguments. Parts 2-5 of the proposition are obtained by straightforward algebraic manipulations.

1. Given other firms' contributions, each firm j's optimization problem is

$$
\min _{r_{j}} r_{j}+\frac{\kappa \alpha_{j}}{2 \sum_{k \in N} r_{k}} \quad \text { subject to } \quad r_{j} \geq \delta .
$$

Karush-Kuhn-Tucker conditions for optimality are given by

$$
\begin{aligned}
1-\frac{\kappa \alpha_{j}}{2\left(\sum_{k \in N} r_{k}\right)^{2}}-\mu_{j} & =0, \\
\mu_{j}\left(r_{j}-\delta\right) & =0, \\
\mu_{j} & \geq 0, \\
r_{j} & \geq \delta .
\end{aligned}
$$

By definition, any strategy profile $\boldsymbol{r}^{*}=\left(r_{1}^{*}, \ldots, r_{n}^{*}\right)$ is a Nash equilibrium if and only if it is a solution to (16)-(19) for $j=1, \ldots, n$. Conditions (16)-(19) ensure that there is at least one firm $i$ such that $r_{i}^{*}>\delta$ and $\mu_{i}=0$. Because, if $r_{j}^{*}=\delta$ for all $j$, we would have $\mu_{j}=1-\frac{\kappa \alpha_{j}}{2 n^{2} \delta^{2}}$ for all $j$. Since $\mu_{j} \geq 0$ for all $j$, this requires that $\delta \geq \sqrt{\kappa \alpha_{j} / 2} / n$ for all $j$, which contradicts with the fact that $\delta<\sqrt{\kappa \underline{\alpha} / 2} / n$, as $\underline{\alpha} \leq \alpha_{j}$ for all $j$. Using (16),

$$
\mu_{i}=1-\frac{\kappa \alpha_{i}}{2\left(\sum_{k \in N} r_{k}^{*}\right)^{2}}=0
$$

Now firm $i$ that satisfies (20) has to belong to the set $L(N)$. Otherwise, for any $k$ with $\alpha_{k}>\alpha_{i}$, we have $\mu_{k}<0$ violating condition (18). Conditions (20) and (16) also show that $\mu_{j}>0$ for all $j \in N \backslash L(N)$. Therefore, using (17), we have, for $j \in N \backslash L(N)$,

$$
r_{j}^{*}=\delta
$$

and, for $j \in L(N)$,

$$
r_{j}^{*} \geq \delta \quad \text { and } \sum_{i \in L(N)} r_{j}^{*}=\sqrt{\frac{\kappa \alpha_{n}}{2}}-(n-\ell) \delta .
$$


The following chain of inequalities show that the conditions on the vector $\left(r_{n-\ell+1}^{*}, \ldots r_{n}^{*}\right)$ are consistent:

$$
\delta<\frac{\sqrt{\kappa \underline{\alpha} / 2}}{n} \leq \frac{\sqrt{\kappa \alpha_{1} / 2}}{n} \leq \frac{\sqrt{\kappa \alpha_{n} / 2}}{n}<\frac{\sqrt{\kappa \alpha_{n} / 2}}{n-1} \leq \frac{\sqrt{\kappa \alpha_{n} / 2}}{n-\ell} .
$$

2. Straightforward from 1.(b).

3. In equilibrium, aggregate contributions from the $n$ firms is $\sum_{i \in N} r_{i}^{*}=$ $\sum_{i \in N \backslash L(N)} r_{i}^{*}+\sum_{i \in L(N)} r_{i}^{*}=(n-\ell) \delta+\sqrt{\kappa \alpha_{n} / 2}-(n-\ell) \delta=\sqrt{\kappa \alpha_{n} / 2}=R_{n}^{d}$. The resulting cycle time is $T_{N}^{g}=\tau_{N}\left(\boldsymbol{r}^{*}\right)=\kappa / \sum_{i \in N} r_{i}^{*}=\kappa \sqrt{\kappa \alpha_{n} / 2}=$ $\sqrt{2 \kappa / \alpha_{n}}=T_{n}^{d}$.

4. Since equilibrium total replenishment cost for the $n$ firms is equal to the aggregate contributions, the claim in 4(a) follows from 3(a) above. The claim in 4(b) results from straightforward substitution and summing over $n$ firms. Part 4(c) is obtained by summing the results in parts (a) and (b) and combining terms.

5. Part 5(a) follows from 1(a) directly for $j \in N \backslash L(N)$. For a firm $j \in L(N)$, we note that his maximum equilibrium contribution is obtained when other firms in $N$ each contribute $\delta$. Part 5(b) follows from substituting the equilibrium cycle time in the expression for $j$ 's holding cost rate. Part 5(c) follows from adding the replenishment and holding costs in parts 5(a) and 5(b).

Equilibrium cycle time depends on the $2 n$-vector $\left(\alpha_{1}, \ldots, \alpha_{n}, \lambda_{1}, \ldots, \lambda_{n}\right)$ of the firms' characteristics only through $\alpha_{n}$-it is invariant to the number of firms and to the finer details of the firms' characteristics as long as $\alpha_{n}$ remains fixed. Similarly, equilibrium total cost depends only on two statistics, namely $\alpha_{n}$ and $\sum_{k \in N} \alpha_{k}$, of the firms' characteristics.

In the absence of a minimum contribution requirement (i.e., if $\delta=0$ ), the order cost is paid by the firms in $L(N)$. If the set $L(N)$ is a singleton, i.e., $L(N)=\{n\}$, in the unique Nash equilibrium, firm $n$ (the firm with the highest stand-alone replenishment rate in $N$ ) pays $\kappa$ per order and incurs a total cost equal to his stand-alone cost. Other firms ride free and enjoy free deliveries. A free-rider's equilibrium payoff is better than his stand-alone payoff since he does not contribute to the ordering cost and the joint cycle time is strictly better than his stand-alone cycle time. When there are multiple firms with the highest stand-alone replenishment rate, we have multiple equilibria. In some of these equilibria, free-riding can be at its extreme-one of the firms in $L(N)$ finances the entire replenishment cost and others ride free. In any equilibrium that involves more than one contributor, all firms are strictly better off compared to independent replenishment.

\section{Comparison of cycle times and aggregate costs}

We can now perform a four-way comparison of cycle times and aggregate total costs under the four modes of joint replenishment: independent, centralized, and non-cooperative joint replenishment under the private contribution game and the direct revelation game studied in MGB. 
As noted above, the equilibrium cycle time depends on the details of the replenishment environment only through $\alpha_{n}$, the maximum of the $n \alpha$ s. Similarly, equilibrium total cost depends only on two statistics, namely $\alpha_{n}$ and $\sum_{k \in N} \alpha_{k}$, of the firms' characteristics. For comparisons of cycle times and aggregate costs we obtain a further simplification. Namely, the comparisons depend on the ratios $\theta_{j}=\alpha_{j} / \sum_{k \in N} \alpha_{k}$, rather than the levels of the parameters. Note that the ordering of these $n$ ratios is the same as that of the $\alpha_{j} s$, that is, $\theta_{n}=\max \left\{\theta_{j}: j \in N\right\}$. Furthermore, $\theta_{n}$ takes values in the interval $[1 / n, 1]$, and the two limits are obtained for $n$ firms with common $\alpha \mathrm{s}$ and for $n=1$, respectively. In particular, $\theta_{n}<1$ for $n \geq 2$.

Straightforward algebraic manipulations yield the following ordering of the cycle times under independent, centralized and non-cooperative replenishment:

$$
T_{1}^{d} \geq T_{2}^{d} \geq \cdots \geq T_{n}^{d}=T_{N}^{g}=T_{N}^{c} / \sqrt{\theta_{n}}>T_{N}^{c}
$$

For comparison of aggregate costs, after similar algebraic manipulations, we get

$$
\begin{aligned}
C_{N}^{d}>\left(\left(\sqrt{\theta_{n}}+1 / \sqrt{\theta_{n}}\right) / 2 \sum_{k \in N} \sqrt{\theta_{k}}\right) C_{N}^{d} & =C_{N}^{g} \\
& =\left(\frac{1}{2}\right)\left(\sqrt{\theta_{n}}+1 / \sqrt{\theta_{n}}\right) C_{N}^{c}>C_{N}^{c} .
\end{aligned}
$$

To explore how the degree of dispersion in firm characteristics affects the ratio of aggregate cost under cooperative replenishment to that under the private contributions game, we observe that the ratio

$$
\frac{C_{N}^{g}}{C_{N}^{c}}=\left(\frac{1}{2}\right)\left(\sqrt{\theta_{n}}+1 / \sqrt{\theta_{n}}\right)
$$

is strictly decreasing in $\theta_{n}$. Thus, for fixed $n$, the ratio is largest when the firms have a common $\alpha$. In this case, the ratio becomes

$$
\frac{C_{N}^{g}}{C_{N}^{c}}=\left(\frac{1}{2}\right)(\sqrt{n}+1 / \sqrt{n})
$$

which increases indefinitely with the number of firms.

Finally we compare the equilibrium cycle times and total cost rates under the private contribution game and the MGB direct revelation game for environments where the MGB game has an equilibrium with full participation. Recall, from (4) above, that full participation under the MGB game requires $\theta_{n}<2 /(2 n-1)$. Under, this restriction, using (4)

$$
T_{N}^{M G B}=\sqrt{2 n-1} T_{N}^{c}=\sqrt{2 n-1} \sqrt{\theta_{n}} T_{n}^{g}>T_{n}^{g}
$$


since $\theta_{n} \geq 1 / n>1 /(2 n-1)$ for $n>1$. The condition for existence of an equilibrium with full participation under the MGB game yields the following upper bound:

$$
\sqrt{2} T_{N}^{g}>T_{N}^{M G B}
$$

To compare the aggregate total cost rates that obtain in the constructive equilibrium of the MGB game and the undominated Nash equilibrium of the private contributions game we use (6) and (23) to get

$$
C_{N}^{M G B}=\frac{n}{\sqrt{2 n-1}} C_{N}^{c}=\frac{n}{\sqrt{2 n-1}} \frac{2}{\sqrt{\theta_{n}}+1 / \sqrt{\theta_{n}}} C_{N}^{g},
$$

Hence,

$$
\frac{C_{N}^{M G B}}{C_{N}^{g}}=\frac{2 n}{\sqrt{2 n-1}} \frac{1}{\sqrt{\theta_{n}}+1 / \sqrt{\theta_{n}}} .
$$

For fixed $n$, the right-hand-side of (24) is strictly increasing in $\theta_{n}$, and, it reaches its minimum and maximum when $\theta_{n}=1 / n$ and $\theta_{n}=2 /(2 n-1)$, respectively. Substituting these values for $\theta_{n}$ and simplifying we get the following bounds:

$$
\frac{2 n}{\sqrt{2 n-1}} \frac{1}{\sqrt{n}+1 / \sqrt{n}}<\frac{C_{N}^{M G B}}{C_{N}^{g}}<\frac{2 \sqrt{2} n}{2 n+1} .
$$

To establish that the lower bound is strictly greater than 1 , we note the fact that $x(n)=\frac{2 n}{\sqrt{2 n-1}} \frac{1}{\sqrt{n}+1 / \sqrt{n}}$ is strictly increasing in $n$ and $x(2)=1.0866$. Finally, taking limits of the lower and upper bounds, we find that as $n$ increases indefinitely, the lower and upper bounds both converge to $\sqrt{2}$. That is, for large $n$, total cost under the direct mechanism studied in MGB is more than $40 \%$ higher than the total cost under the private contribution mechanism. We conclude by noting that the comparisons would be much more dramatic for situations in which the players' adjusted demand shares are more dispersed than condition (4) allows.

\section{Concluding remarks}

A number of important extensions remain to be explored to build an analytical foundation that captures the details of realistic operational management settings. These extensions fall into two broad categories: explorations of alternative mechanisms and alternative models of cost and information structures. Some examples for the first category are alternative mechanisms with various extensive forms (e.g. multiple stages with various information rules; sequential contributions), alternative message spaces (e.g. contribution schedules $r(T)$ stating a firm's contribution as a function of joint cycle time), and alternative outcome functions mapping the firms' messages to the joint cycle time and cost allocation decisions. Extensions along the environment dimension include models that allow minor setup costs, and models that incorporate uncertainty 
and private information on demand and/or holding cost rates or setup costs. In a companion paper, Körpeoğlu et al. (2010), we explore an extension of the current model to study situations where the firms are asymmetrically informed about each other's $\alpha$ values and characterize the Bayesian equilibrium, along with a numerical study that investigates the impact of information asymmetry on equilibrium contributions.

\section{References}

Aksoy Y, Erengüç S (1988) Multi-item models with coordinated replenishments: a survey. Int J Prod Manag 8:63-73

Anily S, Haviv M (2007) The cost allocation problem for the first order interaction joint replenishment model. Oper Res 55:292-302

Bauso D, Giarre L, Presenti R (2008) Consensus in noncooperative dynamic games: a multiretailer inventory application. IEEE Trans Autom Control 53:998-1003

Bergstrom T, Blume L, Varian H (1986) On the private provision of public goods. J Public Econ 29:25-49

Cachon GP, Netessine S (2004) Game theory in supply chain analysis. In: Simchi-Levi D, Wu SD, Shen ZM (eds) Handbook of quantitative supply chain analysis: modeling in the E-business era. Kluwer, Boston pp 13-66

Chinchuluun A, Karakitsiou A, Mavrommati A (2008) Game theory models and their applications in inventory management and supply chain. In: Chinchuluun A, Pardalos PM, Migdalas A, Pitsoulis L (eds) Pareto optimality, game theory and equilibria. Springer, New York pp 833-865

Dror M, Hartman BC (2011) Survey of cooperative inventory games and extensions. J Oper Res Soc 62:565580

Fiestras-Janeiro MG, Garcia-Jurado I, Meca A, Mosquera MA (2011) Cooperative game theory and inventory management. Eur J Oper Res 210:459-466

Harris FW (1913) How many parts to make at once. Fact Mag Manag 10:135-136, 152

Hartman BC, Dror M (2007) Shipment consolidation: who pays for it and how much? Manag Sci 53:78-87

Jans R, Degraeve Z (2008) Modeling industrial lot sizing problems: a review. Int J Prod Res 46:1619-1643

Khouja M, Goyal S (2008) A review of the joint replenishment problem literature: 1989-2005. Eur J Oper Res 86:1-16

Körpeoğlu E, Şen A, Güler K (2010) A competitive game for joint replenishment with information asymmetry. Working paper, Department of Industrial Engineering, Bilkent University, Ankara, Turkey

Leng M, Parlar M (2005) Game theoretic applications in supply chain management: a review. INFOR 43:187-220

Meca A, Garcia-Jurado I, Borm P (2003) Cooperation and competition in inventory games. Math Methods Oper Res 57:481-493

Meca A, Timmer J, Garcia-Jurado I, Borm P (2004) Inventory games. Eur J Oper Res 156:127-139

Minner S (2007) Bargaining for cooperative economic ordering. Decis Support Syst 43:569-583

Zipkin PH (2000) Foundations of inventory management. McGraw-Hill Higher Education, New York 